\title{
ATIVIDADE IMUNOMODULADORA DOS NOVOS DERIVADOS TIAZOLIDÍNICOS GQ-393, GQ-402 e GQ-403 EM PBMC DE PACIENTES COM ESPONDILITE ANQUILOSANTE
}

\author{
M. A. B. CORREIA ${ }^{1}$, V. A. S. SILVA ${ }^{1}$, M. J. B. M. RÊGO ${ }^{1}$, M. C. PEREIRA ${ }^{1}$, D. B.
} SILVA ${ }^{2}$; R. S. Q. ALVES ${ }^{2 ;}$ M. R. GALDINO-PITTA ${ }^{2}$, I. R. PITTA ${ }^{2}$, C. D. L. MARQUES ${ }^{3}$, A. L. B. P. DUARTE ${ }^{3}$, M. G. R. PITTA ${ }^{1}$

${ }^{1}$ Universidade Federal de Pernambuco, Centro de Biociências, Núcleo de Pesquisa em Inovação Terapêutica, Laboratório de Imunomodulação e Novas Abordagens Terapêutica;

2 Universidade Federal de Pernambuco, Núcleo de Pesquisa em Inovação Terapêutica, Laboratório de Planejamento e Síntese de Fármacos;

${ }^{3}$ Universidade Federal de Pernambuco, Hospital das clínicas, Ambulatório de Reumatologia. E-mail para contato: marandrezabcorreia@yahoo.com.br

RESUMO - A espondilite anquilosante (EA) é uma doença reumática crônica inflamatória com alto índice de morbidade entre os pacientes e acometimento principalmente da coluna axial ocasionando perca da mobilidade corporal. $O$ tratamento tradicional da inflamação é feito por uso de anti-inflamatórios nãoesteroidais (AINE), sulfassalazina, metotrexato e agentes inibidores do fator de necrose tumoral (anti-TNF), no entanto sua ação não atua retardando a progressão da doença após iniciada. Para avaliar a ação imunomoduladora foram utilizados novos derivados tiazolidínicos, visto que sua ação vem sendo investigada e relatada na literatura. O objetivo do trabalho foi avaliar a citotoxicidade dos novos derivados tiazolidinicos GQ-393, GQ-394, GQ-396 e GQ-400 GQ-402 e GQ-403 e avaliar a expressão das citocinas IL-17, IL-6, IL23, IL-1,$I F N-\gamma$ e TNF- $\alpha$ sob ação dos derivados tiazolidínicos GQ-393, GQ-402 e GQ-403 no sobrenadante de cultura de pacientes e voluntários sadios. As PBMC de amostras de sangue periférico de pacientes e voluntários sadios foram isoladas, plaqueadas e tratadas nas concentrações 1, 10 e 50mM. Após 48h de tratamento os níveis de IL-17, IL-6, IL-23, IL-1 $\beta$, IFN- $\gamma$ e TNF- $\alpha$ foram quantificados por ELISA. Para as análises estatísticas foi realizado o teste Wilcoxon. $O$ derivado tiazolidínico GQ-403 apresentaram atividade imunomoduladora significativa da IL-1 $\beta$ em voluntários sadios. Em EA os derivados tiazolidínicos diminuíram a secreção de TNF- $\alpha$ e IFN- $\gamma$ comparado ao estímulo. Esses achados podem sugerir um papel imunomodulador desses derivados, sendo necessários estudos adicionais.

Palavras-chave: Citotoxicidade. Citocinas. Atividade anti-inflamatória.

ABSTRACT - Ankylosing spondylitis (AE) is a chronic inflammatory rheumatic disease with a high morbidity rate among patients and mainly affecting the axial 
spine, causing loss of body mobility. The traditional treatment of inflammation is through the use of non-steroidal anti-inflammatory drugs (NSAIDs), sulfasalazine, methotrexate, and tumor necrosis factor (anti-TNF) inhibitors, but their action does not act to slow the progression of the disease after initiation. To evaluate the immunomodulatory action, new thiazolidine derivatives have been used, since their action has been investigated and reported in the literature. The aim of the present study was to evaluate the cytotoxicity of the new thiadiazole derivatives $G Q-393, G Q-394, G Q-396$ and $G Q-400 G Q-402$ and $G Q-403$ and to evaluate the expression of cytokines $I L-23, I L-1 \beta, I F N-\gamma$ and TNF- $\alpha$ under the action of the thiazolidine derivatives $G Q-393, G Q-402$ and $G Q-403$ on the culture supernatant of patients and healthy volunteers. PBMCs from peripheral blood samples from patients and healthy volunteers were isolated, plated and treated at concentrations 1,10 and $50 \mathrm{mM}$. After 48 hours of treatment the levels of IL-17, $I L-6, I L-23, I L-1 \beta, I F N-\gamma$ and TNF- $\alpha$ were quantified by ELISA. Wilcoxon test was performed for statistical analysis. The thiazolidine derivative GQ-403 showed significant immunomodulatory activity of IL-1 $\beta$ in healthy volunteers. In EA, thiazolidine derivatives decreased the secretion of TNF- $\alpha$ and IFN- $\gamma$ compared to the stimulus. These findings may suggest an immunomodulatory role for these derivatives, and further studies are needed.

Keywords: Cytotoxicity. Cytokines. Anti-inflammatory activity.

\section{INTRODUÇÃO}

Espondilite anquilosante é uma doença reumática crônico-inflamatória que afeta predominantemente as articulações do esqueleto axial e muitas vezes as articulações periféricas. As manifestações clínicas mais frequentes são: dor lombar inflamatória, artrite periférica variável, entesite e várias manifestações extra-articulares, incluindo colite, uveíte e psoríase. Com a progressão da doença, as articulações do esqueleto axial inflamado podem se fundir havendo a formação de sindesmófitos (WEISMAN; REVEILLE; VAN-DER HEIJDE, 2006).

Para o diagnóstico são utilizados os critérios de Nova York modificados que consideram a presença de um critério clínico e radiográfico (LINDEN, VALKENBURG, CATS, 1984). Para medir a atividade da doença é aplicado o questionário BASDAI (Bath Ankylosing Spondylitis Disease Activity Index) (TORRES; CICONELLI, 2006). São seis questões que abordam domínios relacionados à fadiga, dor na coluna, dor e sintomas articulares, dor devido ao acometimento das enteses e qualidade e quantidade de rigidez matinal. O escore é medido em escala visual analógica (EVA) de 0 a 10 (0 = bom; $10=$ ruim) $($ ZOCHLING, 2011).

A EA é uma doença cuja etiologia ainda não está totalmente esclarecida, mas se sabe que tem caráter multifatorial, dependendo de fatores ambientais e genéticos. Uma forte associação genética foi demonstrada no antígeno leucócito humano HLA-B27, que é um complexo de histocompatibilidade principal (MHC) classe I (ABDULLAH; YEE, 2015). Os genes dentro da região de MHC, em particular HLA-B27, são responsáveis por quase $25 \%$ de hereditariedade da doença, com pequenas contribuições adicionais de genes de fora do locus MHC, incluindo aqueles que estão envolvidos no processamento de antígenos intracelulares (ERAP1) e genes de citocinas, tais como os envolvidos nas vias IL-17 e IL-23 (REVEILLE, 2012). 
Estudos mostraram que os níveis séricos de TNF- $\alpha$, IL-6, e IL-17A em pacientes com EA, são significativamente mais elevados do que nos controles (YAN et al., 2015). Além disso, as interleucinas IL-17 e IL-22 são citocinas consideradas importantes agentes na condução da inflamação da coluna vertebral e na osteoproliferação (CICCIA et al., 2015). Foram constatados por uma meta-análise níveis séricos elevados de IL-6 e IL-17 nos pacientes (LIU et al., 2015).

O tratamento farmacológico da EA inclui AINES, sulfassalazina, metotrexato e os antiTNF (BRAUN et al., 2011). Embora partes dos pacientes com EA submetidos aos tratamentos tradicionais apresentem melhoria dos sintomas clínicos em longo prazo, uma parcela significativa desses pacientes não responde de forma eficaz ao tratamento convencional (LORENZI et al., 2015; CASO et al., 2015).

$\mathrm{Na}$ busca por novos tratamentos, os derivados dos tiazolidínicos com atividade antiinflamatória reconhecida, podem ser uma escolha promissora para o tratamento de doenças inflamatórias e autoimunes (JUNIOR et al., 2013; SILVA et al., 2013; MALTA et al., 2014). Os derivados tiazolidínicos, são conhecidos por aumentar a sensibilidade a insulina em pacientes portadores da diabetes mellitus tipo 2, além de atividade imunomoduladora (SILVA et al., 2013). Além disso, é de baixo custo, com fácil solubilização, o que permite um maior espectro de aplicações (PANDEY; KUMARI, 2014).

Tais características apontam os derivados tiazolidínicos como moléculas potenciais na modulação imunológica. Com isso, o objetivo do trabalho foi avaliar a citotoxicidade dos novos derivados tiazolidínicos GQ-393, GQ-394, GQ-396 e GQ-400 GQ-402 e GQ-403 e avaliar a expressão das citocinas IL-17, IL-6, IL-23, IL-1 $\beta$, IFN- $\gamma$ e TNF- $\alpha$ sob ação dos derivados tiazolidínicos GQ-393, GQ-402 e GQ-403 no sobrenadante de cultura de pacientes e voluntários sadios.

\section{MATERIAIS E MÉTODOS}

Considerações éticas: $O$ presente trabalho foi aprovado pelo comitê de ética em pesquisa em seres humanos-CEP/CCS/UFPE, sob número CAAE: 55261116.2.0000.5208. Os pacientes e voluntários sadios assinaram o termo de consentimento livre e esclarecido (TCLE).

Compostos derivados de tiazolidínicos: Os compostos GQ-393, GQ-394, GQ-396, GQ-400, GQ-402, GQ-403 foram sintetizados e caracterizados pelo Laboratório de Planejamento e Síntese de Fármacos e apresentaram pesos moleculares: 237.27, 359.83, $403.28,368.45,434.30,404.28$, respectivamente. Os compostos foram inicialmente diluídos na concentração de $50 \mathrm{mM}$ em dimetilsulfóxido (DMSO) e posteriormente de $10 \mathrm{mM}$ em meio RPMI-1640 com 3,7g/L de bicarbonato de sódio, $1 \%$ penicilina e estreptomicina suplementado de $10 \%$ de soro fetal bovino.

Obtenção de células mononucleares de sangue periférico (PBMCs): As PBMC foram separadas com Ficoll, contadas em câmara de Neubauer com o corante azul de Tripan e plaqueadas em placas de 96 poços na concentração de $3 \times 10^{5}$ cél $/ \mathrm{mL}$ para o teste de MTT. O plaqueamento das PBMC dos pacientes e voluntários sadios para o ensaio do ELISA foi realizado em placa de 24 poços na concentração de $10^{6}$ cél $/ \mathrm{mL}$. As células foram cultivadas em meio RPMI-1640 suplementado de $10 \%$ de soro fetal bovino e $1 \%$ de antibiótico. 
Avaliação da atividade citotóxica dos derivados tiazolidínicos: As PBMCs de 9 voluntários sadios foram tratadas com os derivados tiazolidínicos GQ-393, GQ-394, GQ-396 e GQ-400, GQ-402 e GQ-403, nas concentrações de 10, 50, 75 e $100 \mathrm{mM}$. As células foram mantidas em estufa por 48 horas. Posteriormente, foram adicionados $20 \mu \mathrm{L}$ de MTT (3-(4,5dimetiltiazol-2yl)-2,5-difenil brometo de tetrazolina). Após 3 horas de incubação, foi adicionado às placas $130 \mu \mathrm{L}$ de SDS (dodecil sulfato de sódio) a 20\%. As placas foram mantidas em temperatura ambiente por $24 \mathrm{~h}$, protegidas da luz. A viabilidade das células foi determinada por espectrofotometria em leitora de microplacas (Biotek EL808) em 570nm. Os experimentos foram realizados em triplicata.

Determinação da atividade imunomoduladora dos derivados tiazolidínicos: As PBMC de 10 pacientes sadios e 8 pacientes de EA foram cultivadas e tratadas com os derivados GQ-393, GQ-402 e GQ-403 nas concentrações de 1, 10, $50 \mathrm{mM}$ e cultivadas em estufa por 48 horas. Após esse tempo, o sobrenadante foi recolhido e armazenado para posterior dosagem de citocinas.

Determinação de citocinas: As citocinas presentes no sobrenadante de cultura foram quantificadas por teste imunoenzimático ELISA (Enzyme-linked immunosorbent assay) do tipo sanduíche, seguindo as instruções recomendada pelo fornecedor. As citocinas avaliadas foram: IL-6, IFN- $\gamma$ e TNF- $\alpha$ (BD Biosciences) e IL-1 $\beta$, IL-17A e IL-23 (EBiosciences).

Análise dos resultados: As análises estatísticas da citotoxicidade foi realizada em Excel para obter a média e o desvio padrão $(\leq 0,05)$ e os gráficos foram plotados usando o programa GraphPadPrism 5. O teste de Wilcoxon foi utilizado nas comparações dos níveis sérico das citocinas do grupo de pacientes e do grupo controle.

\section{RESULTADOS E DISCUSSÃO}

Para a determinação da concentração das doses dos derivados tiazolidínicos foi avaliada a ação citotóxica através do ensaio de MTT. Estes apresentaram baixa citotoxicidade, com viabilidade de $80 \%$, com exceção do composto GQ-403, que apresentou uma citotoxicidade com viabilidade celular de $78 \%$ na concentração de $100 \mathrm{mM}$ (Figura 1). Determinado o potencial citotóxico e definido as concentrações de trabalho em 1,10 e $50 \mu \mathrm{M}$ dos novos compostos. A metilprednisolona foi utilizada como controle negativo das culturas e inibiu de forma significativa em todas as culturas na dose de $100 \mathrm{mM}$. O Acetato de miristato de forbol (PMA) e Ionomicina (Iono) foram utilizados como estímulo inflamatório nas concentrações de $0,25 \mu \mathrm{g} / \mathrm{mL}$ e $0,2 \mu \mathrm{g} / \mathrm{mL}$, respectivamente. 
Figura 1- Avaliação citotóxica dos derivados tiazolidínicos em células mononucleares de sangue periférico.

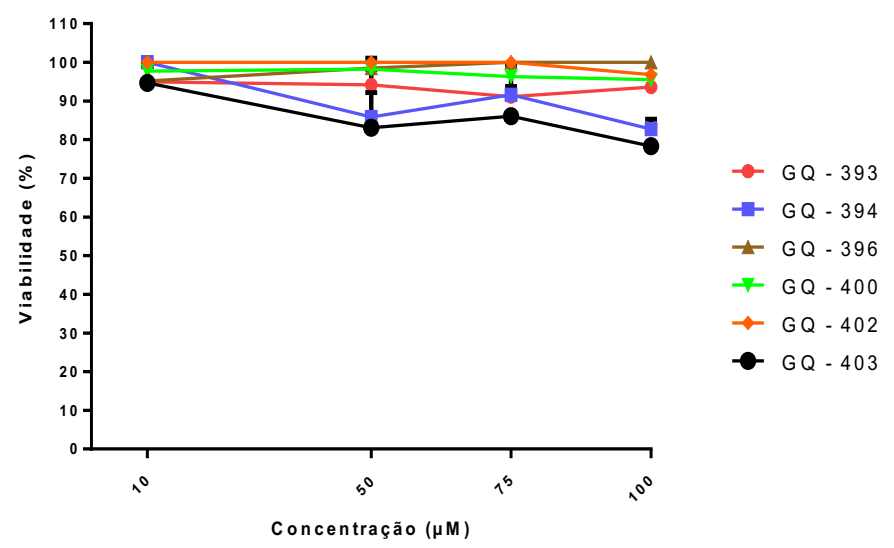

Para avaliar a imunomodulação dos derivados tiazolidínicos, verificando sua possível ação na diminuição de citocinas pró-inflamatórias foi realizado o imunoensaio ELISA. Estudos já constaram o potencial de tratar doenças inflamatórias e autoimunes dos derivados tiazolidínicos, devido à redução da expressão de citocinas como IL-17A, IL-22, IFN-y, em células de pacientes com artrite reumatóide e TNF- $\alpha$ e IL-1 $\beta$ em modelos de ratos com artrite reumatoide (JUNIOR et al., 2013; MALTA et al., 2014).

A citocina IL-1 $\beta$ é uma citocina pró-inflamatória produzida principalmente por macrófagos, monócitos, fibroblastos, células dendríticas, e epiteliais. Estudos apontam que a citocina IL-1 $\beta$ como principal mediadora na perda e erosão óssea em doenças reumáticas, como artrite reumatoide, osteoporose e espondiloartrites, incluindo espondilite anquilosante (PIERO RUSCITTI et al., 2015). A IL-1 $\beta$ apresentou significância na modulação no tratamento com o derivado tiazolidínico GQ-403, comparado ao estímulo PMA/Iono em pacientes saudáveis diminuindo significativamente a expressão de IL-1 $\beta$ nas concentrações de 1,10 e $50 \mu \mathrm{M}$, mediana 567,1;496,1;413,2 pg/mL, p=0,0488; $\mathrm{p}=0,0098 ; \mathrm{p}=0,0059$, respectivamente, comparado com estímulo PMA/Iono $(610,9 \mathrm{pg} / \mathrm{mL}$ ) (Figura 2 D). Os derivados tiazolidínicos não modularam a IL-1 $\beta$ significativamente no grupo de pacientes (Figura $2 \mathrm{C}$ ).

Os derivados tiazolidínicos com suas respectivas medianas: GQ-393 (161,4 pg/mL), GQ-402 (147,1 pg/mL) e GQ-403 (151,4 pg/mL) diminuíram a secreção de TNF- $\alpha$ na concentração de $50 \mathrm{mM}$, quando comparada com o PMA/Iono $(198,9 \mathrm{pg} / \mathrm{mL})$ em paciente EA de forma não significativa (Figura 2 E). Já o composto GQ-393 $(581,7 \mathrm{pg} / \mathrm{mL}$ ) apresentou uma diminuição de IFN- $\gamma$ em pacientes e não nos voluntários sadios em comparação com o PMA/Iono (673,2 pg/mL) na dose de $50 \mathrm{mM}$ não significativamente (Figura 2 A e B). A IL6 e a IL-17A não apresentaram diminuição da expressão com os derivados tiazolidínicos testados e a IL-23 não apresentou expressão (dados não mostrados). 
Figura 2 - Efeito do GQ-393, QG-402 e GQ-403 na inibição de citocina IFN- $\gamma$, IL-1 $\beta$ e TNF$\alpha$ em pacientes com EA e voluntários sadios.
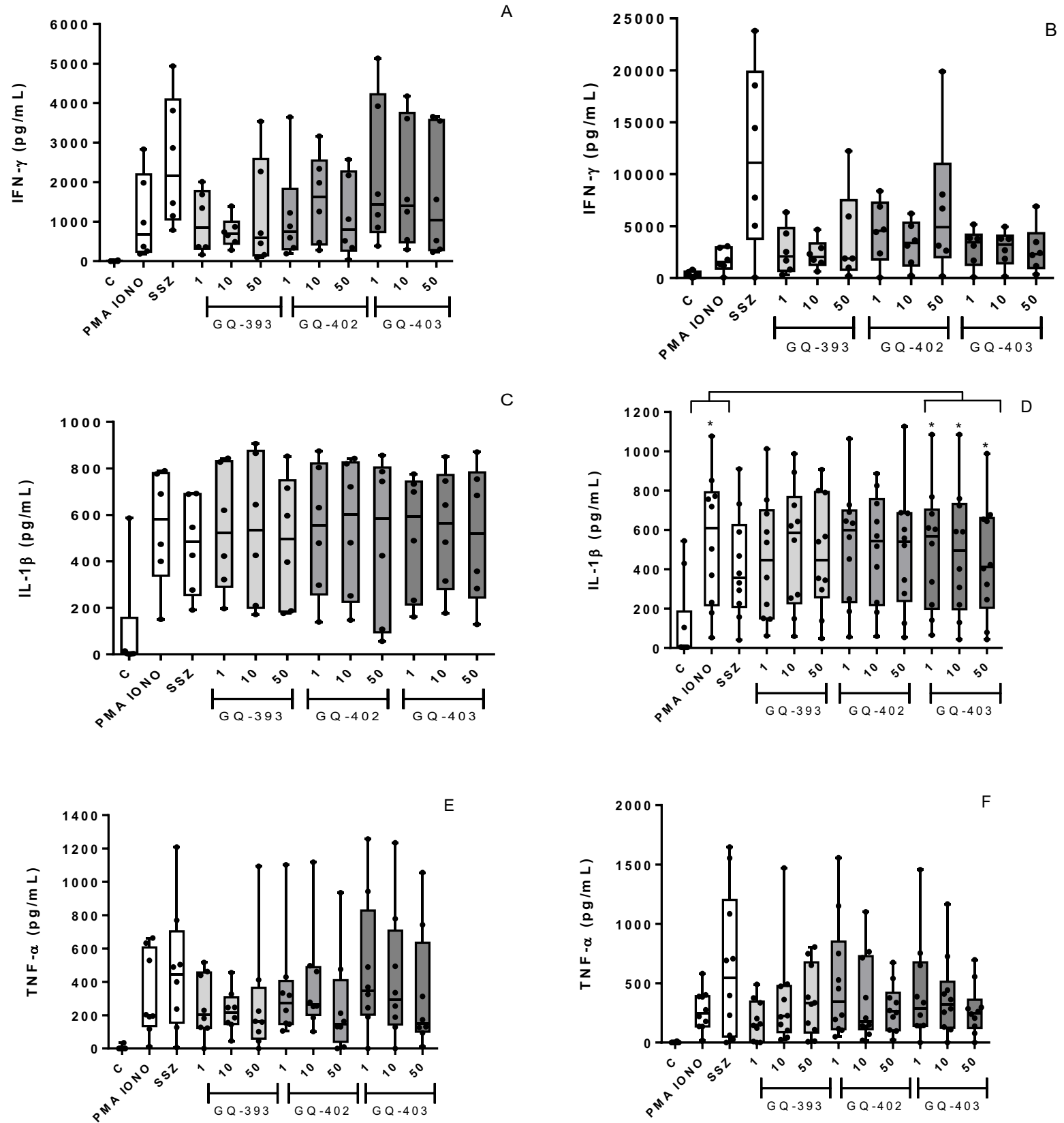

Legenda: PBMC de pacientes e voluntários sadios foram estimulados com PMA/Iono, estimulante inflamatório e tratados em diferentes concentrações dos derivados tiazolidínicos GQ-393, GQ-402 e GQ-403. Grupo de pacientes (A, C e E), e grupo de voluntário sadios (B, $\mathrm{D}$ e F). (*) diferença significativa comparada com o PMA/Iono.

\section{CONCLUSÃO}

Os derivados tiazolidínicos apresentam baixa citotoxicidade, mostrando viabilidade celular acima de $80 \%$ para todos os compostos, com exceção do GQ-403, que apresentou 78\% de viabilidade celular na maior dose testada. No teste imunoenzimático, o composto GQ-403 
apresentou diminuição da IL-1 $\beta$ no sobrenadante de cultura nas condições testadas comparado com o estímulo PMA/Iono dos voluntários sadios, mas não nos pacientes com EA. O GQ-393, GQ-402 e GQ-403 diminuíram a secreção de TNF- $\alpha$ e GQ-393 em IFN- $\gamma$ em pacientes e não nos voluntários sadios.

\section{REFERÊNCIAS}

ABDULLAH, H.; YEE, K. Interação KIR3DL1 com HLA-B27 é alterada por Espondilite Anquilosante Associated ERAP1 e Reforçada por MHC Classe I Cross-linking. Discovery Medicine. v. 20, 2015.

BRAUN, J.; VAN DEN, B. R.; BARALIAKOS, X.; BOEHM, H.; BURGOSVARGAS, R.; COLLANTES-ESTEVEZ, E. et al. 2010 UPDATE OF THE ASAS/EULAR recommendations for the management of ankylosing spondylitis. Ann Rheum Dis.v.70(6), p. 896-904, 2011.

CASO et al., Pharmacological treatment of spondyloarthritis: exploring the effectiveness of nonsteroidal anti-inflammatory drugs, traditional disease-modifying antirheumatic drugs and biological therapies. Ther. Adv Chronic Dis, v. 6, p. 328-338, 2015.

CICCIA, F.; GUGGINO, G. RIZZO, A.; SAIEVA, L.; PERALTA, S.; GIARDINA,A.; CANNIZZARO, A.; SIRECI,G.; DE LEO, G.; ALESSANDRO,R.; TRIOLO, G. Type 3 innate lymphoid cells producing IL-17 and IL-22 are expanded in the gut, in the peripheral blood, synovial fluid and bone marrow of patients with ankylosing spondylitis. Ann Rheum Dis. v. 74, p.1739-1747, 2015.

HAROON et al. Serum Cytokine Receptors in Ankylosing Spondylitis: Relationship to Inflammatory Markers and Endoplasmic Reticulum Aminopeptidase Polymorphisms. J Rheumatol. v. 37, p. 1907-1910, 2010.

JUNIOR et al., Synthesis of a Novel Thiazolidinedione and Evaluation of Its Modulatory Effect on IFN- $\gamma$, IL-6, IL-17A, and IL-22 Production in PBMCs from Rheumatoid Arthritis Patients,

Bio Med Research International, 2013.

LIU; WU; ZHANG; LIU; XUE; WANG; LIU; JIANG; KWANG; WU. Elevated serum levels of IL-6 and IL-17 may associate with the development of ankylosing spondylitis. Int J Clin Exp Med. v.8, p.17362-17376, 2015.

LINDEN, VALKENBURG, CATS. The risk of developing ankylosing spondylitis in HLA-B27 positive individuals. Arthritis \& Rheumatism. v. 27, p. 241-249, 1984.

LORENZI et al., Predictors of response and drug survival in ankylosing spondylitis patients treated with infliximab, BMC Musculoskelet Disord. v. 16, 2015.

MALTA et al., Anti-inflammatory, Anti arthritic and Antinociceptive Activities of 3,5Disubstituted Thiazolidine Derivatives, British Journal of Pharmaceutical Research, Vol. 4, p. 992-1003, 2014.

PIERO RUSCITTI et al., The Role of IL-1 $\beta$ in the Bone Loss during Rheumatic Diseases. Mediators of Inflammation. 2015. 
REVEILLE, J. D. Genetics of spondyloarthritis - beyond the MHC. Nature Reviews Rheumatology. v. 8, p. 296-304, 2012.

SILVA et al., Effect of new thiazolidine derivatives LPSF/GQ-02 and LPSF/GQ-16 onatheroscleroticlesions in LDL receptor-deficient mice (LDLR-/-), Cardiovascular Pathology, Vol. 22, p. 81-90, 2013.

TORRES, T. M.; CICONELLI, R. M. Instrumentos de Avaliação em Espondilite Anquilosante. Rev Bras Reumatol, v. 46, supl.1, p. 52-59, 2006.

WEISMAN, M.H.; REVEILLE, J.D., VAN-DERHEIJDE, D. C. H. 10: Epidemiologia da Espondilite Anquilosante e espondiloartropatias relacionados. Espondilite Anquilosante e espondiloartropatias. Mosby /EIsevier, v.2006, p.117-131, 2006.

YANG; SUNDRUD; SKEPNER; YAMAGATA. TARGETING Th17 cells in autoimmune diseases. Trends in Pharmacological Sciences. v. 35, 493-500, 2014.

ZOCHLING, J. Measures of Symptoms and Disease Status in Ankylosing Spondylitis. Arthritis Care \& Research. v.63, p47-58., 2011.

\section{AGRADECIMENTOS}

Ao Laboratório de Imunomodulação e Novas Abordagens Terapêuticas (LINAT), ao Núcleo de Pesquisa em Inovação Terapêutica (NUPIT), ao Hospital das Clínicas (UFPE) e as agências de fomento CNPQ e FACEPE. 